\title{
Performance Characterization of Heterojunction Bipolar Transistor as an Optoelectronic Mixer
}

\author{
Nur Amirah Shaharuddin ${ }^{a}$, Sevia Mahdaliza Idrus $^{\mathrm{a}}$, Norliza Mohamed $^{\mathrm{a}}$, Abu Bakar ${ }^{\mathrm{a}}$, Suhaila Isaak $^{\mathrm{a}}$ \\ ${ }^{a}$ Faculty of Electrical Engineering, Universiti Teknologi Malaysia, 81310 UTM Johor Bahru \\ *Corresponding author: amirahshah@gmail.com
}

\section{Article history}

Received: 8 March 2012

Received in revised form: 10 April

2012

Accepted: 18 July 2012

Graphical abstract

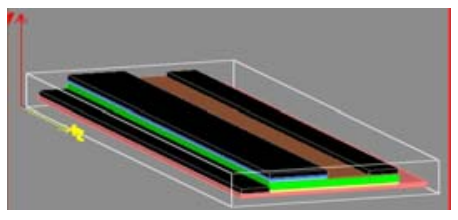

\section{Abstract}

This paper explains the development of physical model Heterojunction Bipolar Transistor (HBT) and characterizes its performance as an optoelectronic mixer (OEM). HBT has been identified as a suitable device to be implemented in optoelectronic mixers by simultaneously photodetecting an intensity modulated laser beam at $1550 \mathrm{~nm}$ and frequency translating the detected signal to a higher or lower frequency which can provide high mixing efficiency and required condition for an oscillator. The HBT OEM was designed, modeled and simulated by using APSYS Crosslight software. Data from the simulation such as the gummel plot, energy band diagram and other characteristics have been generated and analyzed. The device was analyzed considering $1550 \mathrm{~nm}$ wavelength with up to $30 \mathrm{GHz}$ modulating signal frequency. Hence, the designed HBT is found to be possible for the implementation of the broadband RoF system as it can perform the photodetection, amplification and frequency conversion simultaneously as required at RoF remote antenna unit..

Keywords: Heterojunction Bipolar Transistor, Optoelectronic mixer, frequency-up converter, photodetector

(C) 2012 Penerbit UTM Press. All rights reserved.

\subsection{INTRODUCTION}

Millimeter-wave frequencies the are promising RF frequencies for transmitting broadband signal such as video signals and high speed data. Millimeter wave wireless networks should have small cell sizes since millimeter-wave have high transmission loss in air. While the small size cell can offer high frequency reusability and low power consumption, it requires a large number of base stations. Radio over Fiber (RoF) technology is the proposed solution for reducing cost and provides higher reliable communication services which can connect a large number of base stations to one central station trough fiber.

OEM is implemented in RoF system as it performs upconversion and frequency mixing [1]. In the up-conversion scheme with local oscillator (LO), data is transmitted from central station to base station in optical immediate frequency (IF) and frequency is up-converted to the desired radio frequency (RF) with LO signal. The single HBT is one of the most promising optoelectronic converters for RoF communication systems and is applied at OEM as it perform photodetection and low noise frequency [2].

The aim of this paper is to show performance of physical model device of HBT In addition, the characterization of HBT as an OEM to perform up to $30 \mathrm{GHz}$ frequency mixing and performs photodetection that meet $1550 \mathrm{~nm}$ wavelength will be discussed further in this paper.

\subsection{HETEROJUNCTION AS AN OPTOELECTRONIC MIXER}

The HBT similar as bipolar junction transistors (BJT), consists of an emitter, base, and collector. Heterojunction bipolar transistors are bipolar junction transistors, which are composed of at least two different semiconductors. As a result, the energy bandgap as well as all other material properties can be different in the emitter, base and collector. In addition, HBT can handle signals of very high frequencies up to several hundred GHz.

The HBT is common in the modern ultrafast circuits, mostly radio-frequency (RF) systems, as well as RoF requiring a high power efficiency, such as power amplifiers in cellular phones. In addition, it becomes a natural choice for very high frequency military applications requiring a high current drive, high voltage handling capability and low noise oscillator. InP/InGaAs HBTs is aimed for optoelectronic circuits for $1.55 \mathrm{um}$ fiber optic communications. HBT offers a lot of applications instead of other devices such as HEMT and GaAs FETs [7] when implemented in OEM. HBT can reduce phase noise as the optical injection looking achieved with the injection of an optical signal modulated at the oscillation frequency as [9]. In HBT, there are two internal p-n junctions where the mixing process occurs. The process happens when two junctions exhibit nonlinear exponential current-voltage characteristic.

HBT provides several advantages. Instead, it is suitable to implement in OEM. One of the advantages is provide higher frequency conversion [7]. InGaAs HBT can operate over a broad range of frequencies which are required for many high speed, high 
bandwidth applications. Higher operational frequencies enable more data to be transmitted through a fiber or wireless system. HBT improving maximum oscillation frequency, fmax which maximum power gain reach as in (1) is;

$$
f_{\max }=\frac{\sqrt{f_{\tau}}}{\sqrt{8 \pi R_{B} C_{B C}}}
$$

where $\mathrm{RB}$, is the base resistance, $\mathrm{CBC}$, is the base collector capacitance, and $\mathrm{fT}$ is the current gain cutoff frequency.

One of the advantage of HBT is it consist of simple design. HBT only require a single, low-voltage power supply for operation. In addition, HBT consume no power when they are turned off, thereby eliminating the switch components needed for other transistor types. Thus, HBT solutions are easier to design, utilize fewer components and are more compact.

In addition, HBT are not restricted to its de performance. Microwave devices can also be improved dramatically by using an appropriate heterojunction material system. To illustrate this point the transit frequency, $f_{T}$ equation.

$$
\mathrm{f} \tau=\frac{1}{2 \pi \tau}
$$

Where the total transit time equals is,

$$
\tau=\tau_{\mathrm{E}}+\tau_{\mathrm{B}}+\tau_{\mathrm{C}}=\frac{\mathrm{V}_{\mathrm{C}} \mathrm{C}_{\mathrm{BE}}+}{\mathrm{I}_{\mathrm{E}}} \frac{\mathrm{W}_{\mathrm{B}}{ }^{2}+}{2 \mathrm{D}_{\mathrm{n}, \mathrm{b}}} \frac{\mathrm{X}_{\mathrm{d}, \mathrm{BC}}}{2 \mathrm{~V}_{\mathrm{sat}}}
$$

Since a HBT have large current gain, even if the base doping density is higher than the emitter doping density, the base can be much thinner even for the same punch through voltage. Therefore, reduce the base transit time without increasing the emitter charging time, while maintaining the same emitter current density. The transit frequency can be further improved by using materials with a higher mobility for the base layer and higher saturation velocity for the collector layer. In HBT, the largest mixing efficiency achieved when HBT in active mode [5].

The maximum oscillation frequency, fmax in (1), can able further improved. The improved transit frequency immediately increases fmax. The higher base doping also provides a lower base resistance and a further improvement of fmax. As in the case of a homojunction BJT, the collector doping can be adjusted to trade off a lower the collector transit time for a lower basecollector capacitance. The fundamental restriction of heterojunction structures still applies, which the materials must have a similar lattice constant so that they can be grown without reducing the quality of the material.

An equivalent circuit of HBT under optical light can be shown in Figure 1. Photodiode located between base and emitter to absorb the optical light with $1550 \mathrm{~nm}$ wavelength and convert from optical light to electrical energy. An incident light is directed to the optical window of the HBT. Due to the photon absorption of the light, electron-hole pair will be generated and occur in three regions, which are at the base, base-collection depletion and collector regions. They are immediately separated by the strong electric field. Hence, the primary photocurrent, Iprim begins to flow.

The HBT type using is n-p-n junction in which the emitter and the collector are n-type semiconductors, and the base is p-type and operating in common emitter. The two of $\mathrm{PN}$-junctions is baseemitter junction (BE junction) and base-collector junction (BC junction). In the forward active mode the $\mathrm{BE}$ junction is forward biased, while $\mathrm{BC}$ junction is reverse bias.

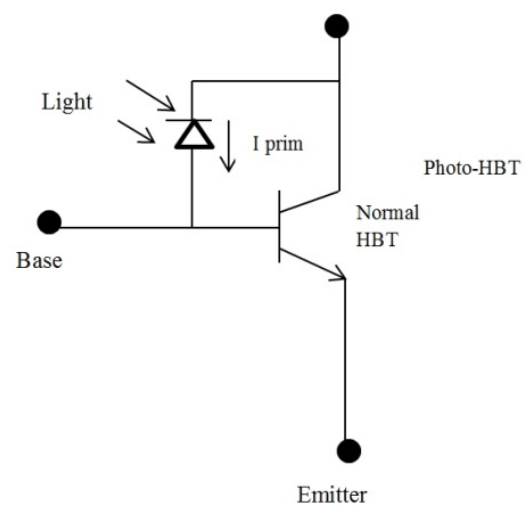

Figure 1 Circuit representation of HBT

\subsection{MODELING-HBT PHYSICAL MODE}

APSYS Crosslight is used to build and modeling the structure of HBT OEM. The layer structure of the three-terminal InP/InGaAs

\begin{tabular}{|c|c|c|c|c|}
\hline & $\begin{array}{c}\text { Sub } \\
\text { collector }\end{array}$ & Collector & Base & Emitter \\
\hline \multirow[t]{2}{*}{ Height $(\mu \mathrm{m})$} & 0.4 & \multirow[t]{2}{*}{0.75} & \multirow[t]{2}{*}{0.05} & \multirow[t]{2}{*}{0.15} \\
\hline & 0.25 & & & \\
\hline \multirow[t]{2}{*}{ Width $(\mu \mathrm{m})$} & 13.00 & \multirow[t]{2}{*}{9.00} & \multirow{2}{*}{9.00} & 4.00 \\
\hline & 9.00 & & & 4.00 \\
\hline Doping Type & $\mathrm{n}$ & $\mathrm{n}$ & $\mathrm{p}$ & $\mathrm{n}$ \\
\hline \multirow[t]{2}{*}{ Material } & InGaAs & \multirow[t]{2}{*}{ InGaAs } & \multirow[t]{2}{*}{ InGaAs } & $\mathrm{InP}$ \\
\hline & $\mathrm{InP}$ & & & InGaAs \\
\hline \multirow{2}{*}{$\begin{array}{l}\text { Concentration } \\
\left(\mathbf{m}^{-3}\right)\end{array}$} & $2 \mathrm{e}+025$ & \multirow[t]{2}{*}{$2 \mathrm{e}+021$} & \multirow[t]{2}{*}{$5 e+025$} & $1 \mathrm{e}+023$ \\
\hline & $1 \mathrm{e}+024$ & & & $1 \mathrm{e}+025$ \\
\hline
\end{tabular}
normal incident HBT is setup following the parameter include in Table1.

Table 1 HBT physical model design parameter

The optical window located at base to minimize the dimension. In addition, it also avoids current crowding effect as the lights are absorbed at base collector junction as the circuit representation in Figure 1 where the photodetector is located at base-collector terminal. The base-emitter junction determines the turn-on voltage of the transistor and the ideality factor of the baseemitter diode. The emitter made a HBT has a wider bandgap than the base and collector region. To improve the gain dependence of optical power or have a larger gain at lower optical power levels is by adjusting the doping of the emitter and base InP. In the base region, a low base resistance can mainly be traded for high current gain. For a high power gain at high frequencies, the base resistance must be small [16]. This can be reached with a high base doping or a large base thickness. Hence, the base doping should be as high as possible which is additionally advantageous for low base contact resistances. The collector width influences the speed properties of the HBT. High speed devices are often designed with a thin collector. With a thicker collector more photons can be absorbed in the depleted region of the collector and therefore the responsivity of the photodiode is improved. 
The design of HBT developed in Apsys in 2D and 3D showed in Figure 2 and Figure 3 as the parameter show in Table 1.

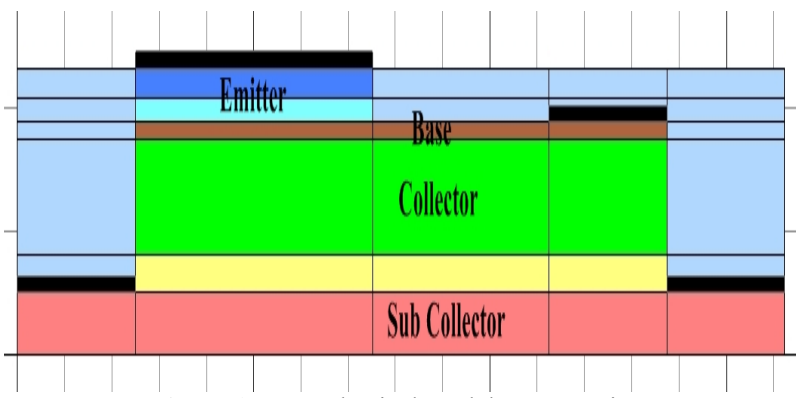

Figure 2 HBT physical model cross section

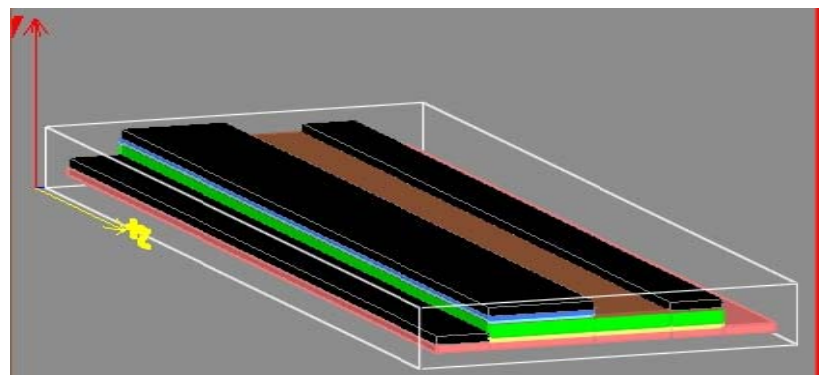

Figure 2 3D HBT model

\subsection{RESULT AND DISCUSSION}

In this part is concentrate to the characterization and analysis of physical model that has been design in APSYS Crosslight. The analysis includes the gummel plot and electric filed against distance.

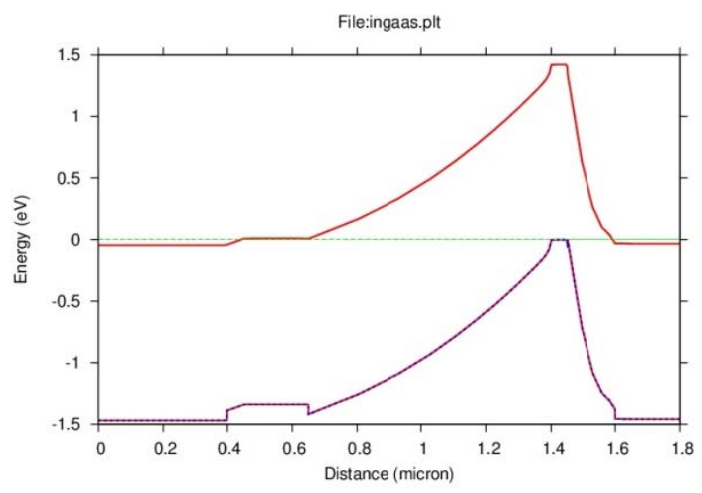

Figure 4 HBT energy band gap

Figure 4 showed energy band diagram versus distance which the upper level of this figure that have plot in red line is conduction band, $E c$ and the lower graph is valance band, $E v$ versus the location was described with a position coordinate or distance in micron. This graph show distance coordinate at $x$ is 4 micron and $y$ from 0 to 1.80 micron where it covers from sub collector to emitter. The band gap difference between emitter and base are larger thus allows a higher efficiency emitter. In addition it leads to forward $V_{B E}$ values. $\Delta E g$ is the difference between the bandgap energy in emitter and band gap energy in base and the value shown $\Delta E g=0 \mathrm{eV}$.It indicates that there is no junction spike at the $\mathrm{InP} / \mathrm{InGaAs}$ interface which is typically behaviour of ordered InP/InGaAs HBT. In addition, the effect of removing the conduction band spike brought about by the conduction band offset. At the InP/InGaAs heterojunction, the larger discontinuity in the valence band as compared to that in the conduction band allows a high injection efficiency without compromising the base doping concentration.

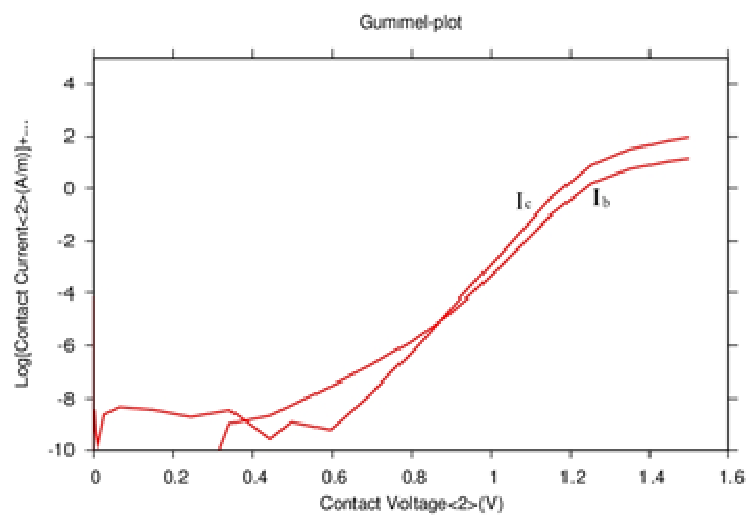

Figure 4 Gummel plot of HBT

Figure 4 shows gummel plot for HBT. Collector current at top curve and base current at bottom curve of a HBT versus the base-emitter voltage. The current due to recombination in the depletion region can be observed as an additional base current between $V_{B E}=0.8 \mathrm{~V}$ and $1 \mathrm{~V}$. The main nonlinear effect in this bias range was the voltage dependence of the dynamic emitter resistance, re which determined the current gain of the HBT at high frequency. As the result show value of $V_{B E}$ is high, re had a small effect on the high frequency ac current gain, because of its small value hence leads to higher amplification level of RF.

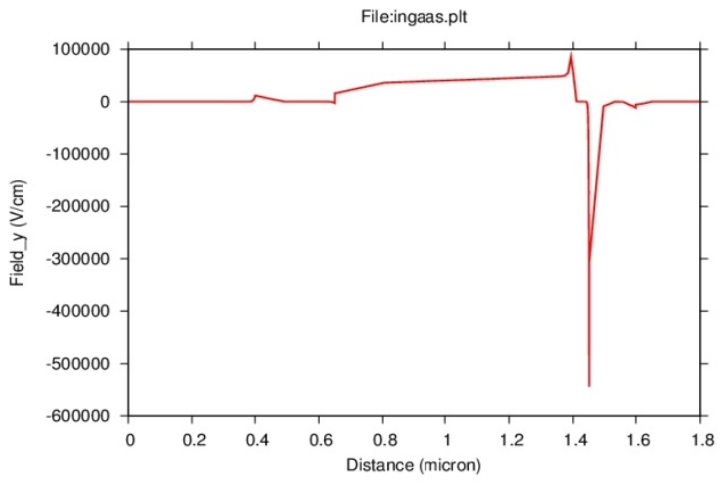

Figure 5 Electric field distribution graph

Peak in Figure 5 showed located between base and collector where electric field corresponding to the depletion region. Basically all the electric field distribution is almost similar but the higher distribution located at between collector and base where it achieve $78903.6 \mathrm{~V} / \mathrm{cm}$ and drop at emitter. In this region, the potential is varying rapidly over a small distance leading high value of electric field. The existence of an electric field requires a significant charge density at each end of the graded regions, which is obtained by a depletion of carriers. This also causes a small cusp in the band diagram. The physical distribution of electric field in HBT is illustrated at figure 6. 


\subsection{CONCLUSION}

This paper has presented a detailed study on the design, modeling and analysis of characterization of HBT as an OEM. We have shown that the physical model of HBT have meet the characterization as an optoelectronic mixer which can be function as photodetector. In addition, HBT is design in small size. Therefore this device can apply in millimeter wave with RoF as it have high frequency mixing operate, can simplified circuit and high efficiency.

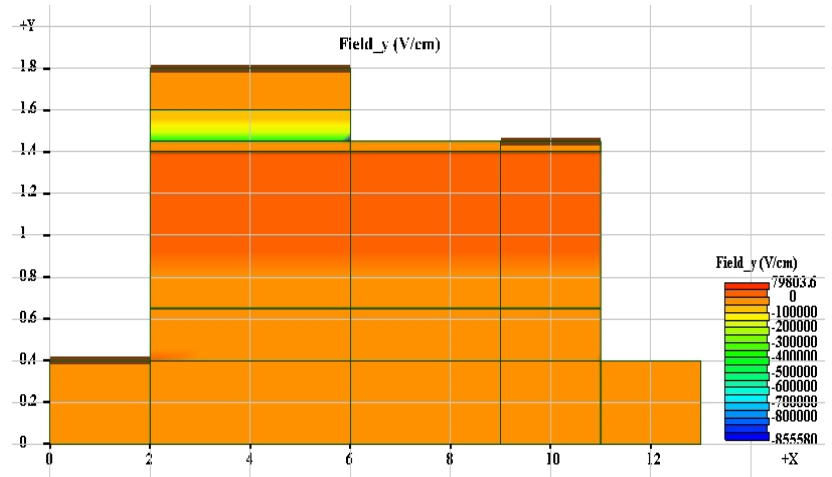

Figure 6 2D Electric field distribution in HBT

\section{Acknowledgement}

The authors acknowledge the Ministry of Higher Education Malaysia and the administration of Universiti Teknologi Malaysia (UTM) for the project financial support through Institutional Grant vote number $02 \mathrm{H} 00$. The gratitude also goes to the Universiti Teknologi MARA for the financial support through the SLAB funding for the main author.

\section{References}

[1] Yoram Betser, Dan Ritter, C. P. Liu, A. J. Seeds, A. Madjar. 1998. A Single-Stage Three-Terminal Heterojunction Bipolar Transistor Optoelectronic Mixer. Journal of Lightwave Technology. Haifa: IEEE. 16: 605 .
[2] Dimitris Pavlidis. 1999. HBT vs. PHEMT vs. MESFET: What's best and why University of Michigan.

[4] Harun, H., S. M. Idrus. 2007. Optical Front-end Receiver Design for Radio over Fiber System. Research and Development, at 5th Student Conference on Research and Development, SCOReD. December 11-12, IEEE: 1 .

[5] Harun, H., S. M. Idrus, N. Liza. 2008. Optical front-end receiver configuration for $30 \mathrm{GHz}$ millimeter-wave signal Radio over Fiber system Circuits and System. In Conference on IEEE Asia Pacific Circuits and Systems APCCAS. 1248.

[6] J. Lasri, Y. Bester, V. Sidorov, S. Cohen, D. Ritter, M. Orenstein, G. Eisenstein. 1999. HBT Optoelectronic Mixer At Microwave Frequency: Modeling And Experimental Characterization in Proc. Journal of Lightwave Techno. 17(8): 1423-1428.

[7] Honjo, K. Applications of HBT' in Solid-State Electronics. 38(9): 15691573.

[8] Tan, S.W, W.T. Chen. 2008. Optical and electrical characteristics of InGaP/AlGaAs/GaAs composite emitter heterojunction bipolar/phototransistors (CEHBTs/CEHPTs) Superlattices and Microstructures. 33(4): 209-216.

[9] Harun, H., S. M. Idrus, N. Liza 2008. HBT Optoelectronic Mixer Design for Radio over Fiber System. International Symposium of High Capacity Optical Networks and Enabling Technologies HONET.

[10] S. M. Idrus, R. J. Green. 2004. Performance Analysis of the Photoparametric Upconverter Using Harmonic Balance Technique' in Proceedings of the 9th IEEE High Frequency Postgraduate Student Colloquium. September 6-7. Coventry, UK: IEEE 23-28.

[11] Mohamed, N. Idrus, S. M. Mohammad, A. B. Harun, H, 2008. Photonic up-conversion frequency utilizing harmonic balance simulation analysis' in on International Conference of Photonics (ICP) 2010. 5-7 July. 1-4.

[12] Mohamed, N., S. M. Idrus. 2008. Review on system architectures for the millimeter-wave generation techniques for RoF communication link. IEEE International RF and Microwave Conference RFM UTM KL. 326.

[13] Takanashi, Y, Fukano, H. 1998. Low-frequency noise of InP/InGaAs heterojunction bipolar transistors. IEEE Transactions on Electron Devices. 45(12): 2400-2406.

[14] Liou, J. J, Drafts, W Yuan, J. 1989. Modeling the heterojunction bipolar transistor for integrated circuit simulation University/Government/Industry Microelectronics Symposium. 12-14 June Proceedings. 8: 219-222.

[15] López-González, J. M. and L. Prat. 1996. Numerical modelling of abrupt InP/InGaAs HBTs. Solid-State Electronics. 39(4): 523-527.

[16] Chau, H. F., D. Pavlidis, et al. 1992. Analysis of InP/InGaAs single and double heterostructure bipolar transistors for simultaneous high speed and high breakdown operation Indium Phosphide and Related Materials. Fourth International Conference.

[17] Hayes, J. R., F. Capasso. 1983. Elimination of the emitter/collector offset voltage in heterojunction bipolar transistors. Electron Devices Meeting, International.

[18] Niu, G. and J. D. 1999. Cressler The impact of bandgap offset distribution between conduction and valence bands in Si-based graded bandgap HBT's.' Solid-State Electronics. 43(12): 2225-2230. 